\title{
$\$$ Research Square

\section{Mitophagy eliminates the accumulation of SARM1 on the mitochondria, alleviating programmed axon death in acrylamide neuropathy}

Shuai Wang ( $\square$ shuaiw@mail.sdu.edu.cn )

Shandong University Cheeloo College of Medicine https://orcid.org/0000-0003-3533-8565

Hui Yong

Shandong University Cheeloo College of Medicine

Cuiqin Zhang

Shandong University Cheeloo College of Medicine

Kang Kang

Shandong University Cheeloo College of Medicine

Mingxue Song

Shandong University Cheeloo College of Medicine

Yiyu Yang

Shandong University Cheeloo College of Medicine

Zhengcheng Huang

Shandong University Cheeloo College of Medicine

Shu'e Wang

Shandong University Cheeloo College of Medicine

Haotong Ge

Shandong University Cheeloo College of Medicine

\section{Xiulan Zhao}

Shandong University Cheeloo College of Medicine

\section{Fuyong Song}

Shandong University Cheeloo College of Medicine https://orcid.org/0000-0003-2662-8210

\section{Research Article}

Keywords: Acrylamide, Axon degeneration, Neurotoxin, Peripheral neuropathy, Rapamycin, Wallerian degeneration,

Posted Date: March 9th, 2022

DOI: https://doi.org/10.21203/rs.3.rs-1173131/v2 
License: (c) (i) This work is licensed under a Creative Commons Attribution 4.0 International License. Read Full License 


\section{Abstract}

Sterile-a and toll/interleukin 1 receptor motif containing protein 1 (SARM1) is the defining molecule and central executioner of programmed axon death (also known as Wallerian degeneration). Although it has been confirmed to have a mitochondrial targeting sequence and can bind to and stabilize PTEN-induced putative kinase 1 (PINK1) for mitophagy induction, deletion of the mitochondrial localization sequence disrupts SARM1 mitochondrial localization in neurons but does not alter its ability to promote axon degeneration after axotomy. The biological significance for mitochondrial localization of SARM1 remains elusive. Here, we demonstrated that the SARM1-dependent axonal destruction pathway was involved in acrylamide (ACR) neuropathy in vivo and in vitro, a moderate Wallerian-like programmed axonal death process. The up-regulated SARM1 accumulated on mitochondria, which interfered with mitochondrial dynamics and activated PINK1-mediated mitophagy. Importantly, rapamycin (RAPA) intervention eliminated mitochondrial accumulated SARM1 and partly attenuated ACR neuropathy. Thus, mitochondrial localization of SARM1 contributes to its clearance through the SARM1-PINK1 mitophagy pathway and mitophagy, in turn, negative feedback inhibits axonal degeneration. Mitochondrial localization of SARM1 is complementary to the coordinated activity of the pro-survival factor, nicotinamide mononucleotide adenyltransferase 2 (NMNAT2), and SARM1, and is part of the self-limiting molecular mechanisms of programmed axon death.

\section{Highlights}

- SARM1-dependent axonal destruction pathway is involved in ACR neuropathy.

- The up-regulated SARM1 accumulates on mitochondria in ACR neuropathy.

- ACR induces mitophagy and the SARM1-PINK1 pathway is one of the molecular mechanisms.

- RAPA intervention clears mitochondria SARM1 and partly attenuates ACR neuropathy.

- Mitophagy clearance of SARM1 is complementary to the coordinated activity of NMNAT2 and SARM1.

\section{Introduction}

Axon degeneration and loss are common hallmarks of neuropathies, traumatic injury, and multiple neurodegenerative disorders. The molecular mechanism has been primarily elucidated through the study of programmed axon death (also known as Wallerian degeneration). Wallerian degeneration, first described by the British neurophysiologist Augustus Waller in 1849 [1], refers to rapid axonal fragmentation after a long period of relative latency due to a genetically encoded self-destruction program that is activated distal to the point of the axon cut site [2-4]. This is the most extreme and typical manifestation of axon death. Over the past decades, great progress has been made in the understanding of this active process. The coordinated activity of both the pro-survival factors and the pro-degeneration factors, exemplified by nicotinamide mononucleotide adenyltransferase 2 (NMNAT2) and sterile-a and toll/interleukin 1 receptor motif containing protein 1 (SARM1), limits degeneration signal 
in an "off" state in healthy axons. After axotomy, NMNAT2 is rapidly consumed in the axon segment due to the interruption of axon transport and the degradation $[5,6]$, resulting in the hindrance of nicotinamide adenine dinucleotide (NAD+) synthesis and storing of nicotinamide mononucleotide (NMN), the precursor of NAD+. The raised ratio of NMN and NAD+ [7] activates SARM1, which further consumes NAD + to switch into the irreversible stage accompanied by adenosine triphosphate (ATP) depletion, neurofilament hydrolysis, and axon fragmentation [8].

SARM1 is the defining molecule of programmed axon death. Expression and activation of SARM1 triggers metabolic catastrophe and axon destruction, whereas genetic deletion and even reduced SARM1 expression protects axons under diverse neurotoxic triggers $[9,10]$. As the central executioner of axon degeneration, SARM1 is evolutionarily highly conserved, having homologues in mouse, Drosophila, zebrafish, Caenorhabditis elegans, amphioxus, and horseshoe crab [11-14]. These homologues share a common domain architecture constituted of autoinhibitory $\mathrm{N}$-terminal armadillo motifs (ARM), tandem sterile a motif (SAM) domains that mediate constitutive homomultimerization, and a C-terminal toll/interleukin 1 receptor (TIR) domain. The N-terminal SAM-TIR domain has NAD + cleaving activity, and the activation induces axonal NAD + depletion. So far, although we have a preliminary understanding of these domains, all of which are involved in protein-protein interactions, the regulatory mechanisms of SARM1 still need to be further studied.

Currently, it is believed that elucidating the exact subcellular localization of SARM1 will offer insights into its regulatory pathways. Even though it remains to be defined, mitochondrial localization has been proposed. The N-terminal 27 amino acids of SARM1 are hydrophobic polybasic and have the capacity to fold into an a-helix that is required for association with the mitochondrial outer membrane. It serves as a mitochondria targeting sequence, associating SARM1 to the mitochondria [15]. The N-terminal region of SARM1 that contains the ARM domain but not the SAM and TIR domains is also shown to be necessary for interaction with PTEN-induced putative kinase 1 (PINK1), a key molecular involved in mitophagy and neuroprotection against various stresses [16]. SARM1 can recruit tumor necrosis factor receptorassociated factor 6 (TRAF6) into SARM1-PINK1 complexes and promotes K63-linked ubiquitination of PINK1 at Lys-433 by TRAF6. And the ubiquitination at the cytosolic face of the outer membrane prevents PINK1 from translocation into the mitochondrial intermembrane space, and is linked to multiple steps of mitophagy. However, previous studies also show that Wallerian degeneration followed by axotomy is only modestly influenced by mitochondria [17]. Deletion of the mitochondrial localization sequence disrupts SARM mitochondrial localization in neurons but does not alter its ability to promote axon degeneration after axon cut [18]. Thus, the biological significance of SARM1 mitochondrial localization has yet to be further explored.

To identify situations that would benefit from blocking SARM1-dependent axonal destruction pathway, a variety of peripheral neuropathies are re-examined. We are committed to this and focus on acrylamide (ACR) neuropathy here. 
As the vinyl monomer for the production of flocculant polyacrylamide, ACR is widely utilized in a variety of industrial settings and laboratories [19]. In addition to occupational exposure, ACR in food, drinking water, coffee, and cigarette smoke has a potential hazard to the general population [20-23]. The potential adverse neurological effects are noted among individuals with high daily exposure to ACR $[24,25]$ and chronic ACR intoxication induces similar peripheral neuropathy in people and animals [26, 27], which are characterized by progressive axon death of the distal ends of the longest and the largest nerve fibres. As exposure continues, progressive retrograde destruction of these distal axon regions ensues with preservation of more proximal segments resulting symptoms, that are, ataxia, skeletal muscle weakness and numbness of the hands and feet. The specific spatiotemporal pattern of axon damage is similar to the profile of Wallerian degeneration after axotomy [28-32] and is named as Wallerian-like degeneration. Studying the changes of SARM1 in such a slowly progressing and moderate axon destruction process will contribute to the strengthening of the understanding of programmed axon death and explore its potential regulatory mechanism. Here, we want to explore whether the SARM1-dependent axonal destruction pathway is involved in peripheral nerve damage in ACR neuropathy? Further, is the mitochondrial SARM1-PINK1 pathway for mitophagy a target for therapeutic intervention in ACR poisoning?

\section{Materials And Methods}

\subsection{Animals and treatments}

Adult male Wistar rats (160-180g, SPF) were supplied by Jinan Pengyue Laboratory Animal Breeding Co., Ltd., Jinan, China. All animals were kept in a barrier system. Food and drinking water were available. The animal room was maintained at approximately $22^{\circ} \mathrm{C}$ and $50 \%$ humidity with a $12 \mathrm{~h} \mathrm{light/dark} \mathrm{cycle.}$ After seven days of acclimatization, rats were randomly divided into groups for experiments.

ACR intoxication at $1-50 \mathrm{mg} / \mathrm{kg}$ per day produces a triad of neurological deficits $[33,34]$ and the noobserved-adverse-effect level (NOAEL) of ACR is determined to be $0.2-0.4 \mathrm{mg} / \mathrm{kg}[35,36]$. With references to ACR intoxication regimens and the extrapolation factor of 100 in toxicology studies, the in vivo ACR neuropathy model set up four groups of control, low, medium, and high doses, 0, 10, 20 and $40 \mathrm{mg} / \mathrm{kg}$ b.w. i.p. every other day, respectively. Rapamycin (RAPA) intervention experiment has the same ACR dose of the high dose group. The effective dose of RAPA to activate autophagy is $0.5-4 \mathrm{mg} / \mathrm{kg}[37,38]$ and 1 $\mathrm{mg} / \mathrm{kg}$ b.w. i.p. once per week is employed here to avoid potential detrimental effects [39].

ACR was dissolved in saline and the control group was treated with same volume of saline. RAPA was dissolved in DMSO, sequentially added with PEG300 and Tween-80 to help dissolve, and diluted with saline (volume ratio: DMSO 2.5\%, PEG300 10\%, Tween-80 1.25\%, saline 86.25\%) to get a clear liquid. The intervention group was given RAPA every Monday and received ACR half an hour later.

\subsection{Neurobehavioral and neurophysiological tests}

\subsubsection{Rotarod latency test}


ZS-ROM rotarod fatigue equipment (Beijing Zhongshidichuang Technology and Development Co., Ltd., Beijing, China) was utilized. All rats received training before intoxication, that was, staying on the equipment for $60 \mathrm{~s}$ at a velocity of $8 \mathrm{rpm}$. During the formal test, the original velocity was set at $0 \mathrm{rpm}$ and accelerated smoothly to $40 \mathrm{rpm}$ within $200 \mathrm{~s}$. The time that animals stayed on the rod was recorded as its latency to fall $[40,41]$.

\subsubsection{Landing foot splay measuring}

Landing foot splay distance was the distance between the inner surfaces of the fourth digits of each foot after the animals were dropped from a $30 \mathrm{~cm}$ height [42].

\subsubsection{Gait score evaluation}

Rats were positioned in an open field and were observed for $3 \mathrm{~min}$. Following the observation, a gait score was assigned from 1 to 4 where 1 = a normal, unaffected gait; 2 = a slightly abnormal gait (tiptoe walking, hindlimb adduction); 3 = moderately abnormal gait (obvious movement abnormalities characterized by dropped hocks and tail dragging); 4 = severely abnormal gait (dragging hindlimbs and complete absence of rearing) $[43,44]$.

\subsubsection{Motor nerve conduction velocity measurement}

The motor nerve conduction velocity of the rat tail was measured with a BL-420E biological function experimental system (Chengdu Taimeng Technology Co., Ltd., Chengdu, China). The electrodes used in our experiments were stainless steel needles, $0.34 \mathrm{~mm}$ in diameter and about $15 \mathrm{~mm}$ long. The rat was fixed in the supine position and its tail was exposed to a pair of stimulating electrodes, which were connected to the two pairs of sensor electrodes, and an earth electrode. A single electric stimulus of $5 \mathrm{~V}$ was applied through the stimulating electrodes and two action potential oscillogram curves were recorded. The time between the two peak points and the distance between the two negative sense electrodes was recorded to calculate the motor nerve conduction velocity [45].

\subsection{Cell culture and treatments}

Mouse Neuro2a (N2a) neuroblastoma cells, purchased from American type culture collection (ATCC, USA), were cultured in high-glucose Dulbecco's Modified Eagle's medium (DMEM) supplemented with $10 \%$ (v/v) fetal bovine serum (FBS), 1\% penicillin-streptomycin, 1\% MEM Non-Essential Amino Acids (NEAA) and $1 \%$ sodium pyruvate at $37^{\circ} \mathrm{C}$ in a $5 \% \mathrm{CO} 2$ incubator. For differentiation, cells were induced in DMEM medium supplemented with $2 \%(\mathrm{v} / \mathrm{v})$ FBS and $10 \mu \mathrm{M}$ retinoic acid for $48 \mathrm{~h}$. Then, differentiated cells were treated with ACR.

\subsection{Cytotoxicity test and cellular morphology}

Cytotoxicity test was conducted by Cell Counting Kit-8 (CCK8) (Tecan Infinite® M200 Pro). Axons of ACR treated N2a cells were observed by microscope (Nikon ECLIPSE Ti). In each group, we observed 10 fields and recorded the length of six axons by TCapture at least for each field. Differentiated N2a cells began to show axon damage after being treated with $0.5 \mathrm{mM} \mathrm{ACR}$ for $24 \mathrm{~h}$, and had obvious axon degeneration 
after being treated with $2.0 \mathrm{mM}$ ACR for $24 \mathrm{~h}$. Based on the results of the cytotoxicity test and cellular morphology, a maximum concentration of $2 \mathrm{mM} \mathrm{ACR}$ was used in this study to ensure that the axondamaged cells had more than $75 \%$ viability compared with the control.

\subsection{LC3 turnover experiment}

Cells were seeded in six-well plates, adhered, differentiated, and pre-treated with $10 \mu \mathrm{g} / \mathrm{mL}$ Pepstatin A and E64d for $1 \mathrm{~h}$ before treatment with $1.5 \mathrm{mM} \mathrm{ACR}[46,47]$.

\subsection{Pathological examination}

\subsubsection{Histopathological examination}

Rats were anesthetized with a 1:1 mixture of $5 \%$ chloral hydrate and $12.5 \%$ urethane. After infusion of saline and $4 \%$ paraformaldehyde solution, the tissues were quickly dissected and separated. Spinal lumbosacral enlargements (L2-S3) were fixed in 4\% paraformaldehyde for $48 \mathrm{~h}$, dehydrated in alcohol, and then embedded in paraffin. Every 20th cross-section $(5 \mu \mathrm{m})$ was processed by haematoxylin and eosin (H\&E) and Nissl staining (0.5\% Thionine solution), scanned as digital slices through Olympus VS120, and analyzed blindly. Cells with a distinct nucleus and a diameter of at least $25 \mu \mathrm{m}$ located in the anterior horn ventral to the line tangential to the ventral tip of the central canal were considered to be a motor neurons. These a motor neurons with abnormal morphological changes, such as hyperchromatic cytoplasm, were manually counted.

\subsubsection{Immunofluorescence staining}

$\mathrm{N} 2 \mathrm{a}$ cells were seeded on sterile cover glasses placed in the 24-well plates. After desired treatment, N2a cells adherent on glasses were washed with phosphate buffered solution (PBS) twice. Then, cells were fixed in $4 \%$ paraformaldehyde solution for $10 \mathrm{~min}$, quenched by $50 \mathrm{mM} \mathrm{NH}_{4} \mathrm{Cl} / \mathrm{PBS}$ for $10 \mathrm{~min}$, permeabilized by $50 \mu \mathrm{g} / \mathrm{ml}$ Digitonin/PBS for $5 \mathrm{~min}$, and blocked by $1 \%$ Gelatin/PBS for 30 min at $4^{\circ} \mathrm{C}$. Between each step, the glasses were washed by PBS. Then, cells were stained with antibodies dilutions $(1: 400)$ at $4^{\circ} \mathrm{C}$ overnight. After washing with PBS, cells were incubated with goat secondary antibody dilutions (1:1000) at room temperature for $1 \mathrm{~h}$. Finally, cells were observed by a Nikon fluorescence microscope. Paraffin tissue sections were dewaxed, hot fixed, and stained as mentioned above. Fiji Image-J was utilized to perform co-localization analysis of fluorescence images, including the Pearson's $R$ value calculation of the entire image and gray intensity analysis of the region of interest (ROI).

\subsubsection{Transmission electron microscopy ultrastructure analysis}

After perfusion, spinal cord (L2-S3) and sciatic nerve (sciatic notch-popliteal fossa) were separated, trimmed to the appropriate size in $2.5 \%$ glutaraldehyde droplets, and fixed. Following ultramicrotomy, the sections were transferred on to a nickel grid and stained with osmium tetroxide, uranyl acetate and lead citrate. The samples were subsequently observed using a JEM1010 transmission electron microscope (Jeol). Fiji Image-J was used for image analysis, including manual identification, automatic selection, 
and measurement of axons and mitochondria. The distributions of axonal diameter and mitochondrial length were analyzed and visualized by GraphPad Prism 8.

\subsubsection{Live cell imaging of N2a cells}

Mito Tracker Green FM staining was applied to visualize mitochondria and Lyso Tracker Red DND-99 to mark lysosomes and other acidic organelles. Hoechst 33342 was used to stain nuclei. Following the user guide, Mito Tracker Green FM and Lyso Tracker Red DND-99 were dissolved in high-quality, anhydrous DMSO to prepare a $1 \mathrm{mM}$ stock solution and were stored at $-40^{\circ} \mathrm{C}$. The Stock solutions of Mito Tracker Green FM and Lyso Tracker Red DND-99 were diluted with DMED to a final concentration of $100 \mathrm{nM}$ and $50 \mathrm{nM}$, respectively. N2a cells treated with $1.5 \mathrm{mM}$ ACR for $24 \mathrm{~h}$ or not were incubated with this working solution for $30 \mathrm{~min}$, and then imaged by a fluorescence microscope.

\subsection{Western blotting}

\subsubsection{Mitochondrial fractionation}

Mitochondria were isolated using a Mitochondria Isolation Kit by sequential centrifugation. Briefly, samples were homogenized and were lysed in Mitochondria Isolation Solution supplemented with protease inhibitor cocktail and phosphatase inhibitor cocktail. Lysates were centrifuged at $1,000 \mathrm{~g}$ for 5 min to remove the plasma membrane fraction, and subsequently, the supernatants were centrifuged at $3,500 \mathrm{~g}$ for $10 \mathrm{~min}$ to get purified mitochondria. The supernatant at this time was the mitochondriadepleted cytoplasmic fraction, and the pellet was the mitochondrial fraction. Given the low yield of mitochondrial fraction and consistency changes of Wallerian degeneration related molecular in the spinal cord and sciatic nerve, we did not extract the mitochondrial components of the sciatic nerve homogenate.

\subsubsection{Samples preparation, electrophoresis, and immunoblotting}

The sciatic nerve was ground into powder in liquid nitrogen and the immunoblotting sample was prepared according to the subsequent steps. The spinal cord was homogenized directly in ice-cold RIPA buffer supplemented with protease inhibitor cocktail and phosphatase inhibitor cocktail. Then, homogenates were centrifuged at $12,000 \mathrm{~g}$ for $10 \mathrm{~min}$. Supernatants were used for immunoblotting analysis. After protein concentration was determined by $\mathrm{BCA}^{\mathrm{TM}}$ Protein assay Kit, the sample was mixed with $4 x$ loading buffer, and then heated at $100^{\circ} \mathrm{C}$ for 5 min.

To assess relative changes in protein content, corresponding protein samples were subjected to sodium dodecyl sulfonate-polyacrylamide gel electrophoresis. Following electrophoresis, proteins were electrophoretically transferred to polyvinylidene fluoride membranes. Then the membranes were blocked with $3 \%$ fat-free milk for 45 min and incubated with primary antibody (Supplementary file 1. Reagent) diluted in $0.1 \%$ BSA for $8 \mathrm{~h}$. Following primary antibody, membranes were washed in a mixture of Tween 20 and tris-buffered saline and incubated with horseradish peroxidase-conjugated secondary antibody at room temperature for $1 \mathrm{~h}$. After being washed again, the membranes were incubated by using the 
SuperSignal West Pico Chemiluminescent Substrate reagents for 2 min and then exposed to Tanon-5200 Multi Chemiluminescence Imaging System (Tanon Science \& Technology, Shanghai, China). Digitized data were quantified as integrated optical density using Fiji Image-J. Each protein was repeated at least three times. VDAC and $\beta$-actin were detected as loading control for mitochondrial proteins and total proteins respectively.

\subsection{Statistical analysis}

The performers were blinded to the experimental design in data collection and analysis. Data are presented as means \pm standard deviation (SD) through GraphPad Prism 8 after analysis using SPSS 18.0 software. Two-way Repeated Measures ANOVA was used for neurobehavioral data. Unpaired t test, oneway ANOVA and two-way ANOVA followed by Bonferroni's post-hoc test, were performed in the right situations. The differences were considered significant at $p<0.05$.

\section{Results}

\subsection{SARM1-dependent axonal destruction pathway is involved in ACR neuropathy}

Exposure to $\operatorname{ACR}(0,10,20$ and $40 \mathrm{mg} / \mathrm{kg}$ b.w. i.p. every other day for four weeks) induced peripheral neuropathy in rats in a time- and dose-dependent manner (Fig. S1A, Fig. 1A). Rats in the high-dose group $(\mathrm{H})$ developed neurobehavioral symptoms two weeks after exposure, and produced a triad of neurological deficits after four weeks of exposure, that was, shorter rotarod staying time, rose landing foot splay distances, and increased gait scores. After four weeks of intoxication, slight gait abnormality occurred in the medium-dose group ( $M)$, while no significant changes were seen in the low-dose group (L) compared with the control (C). Consistent with neurobehavioral indicators, histopathological changes appeared in rats subjected to ACR for four weeks (Fig. 1B-D). Compared with the control, the sciatic nerve axons in rats exposed to ACR were lost. The retained axons were swollen with an increased diameter and myelin sheaths were loose (Fig. 1B, 1C). The a motor neurons in the lateral anterior horn of the spinal cord, innervating transarticular extrafusicular muscle fibres through axons in the sciatic nerve, also showed obvious morphological conversions. The number of abnormal neurons with hyperchromatic cytoplasm increased (Fig. 1B, 1D, Fig. S1B). Furthermore, the motor nerve conduction velocity slowed down in a dose-dependent manner four weeks after poisoning (Fig. 1E). Then, Wallerian degeneration-related molecules were detected by Western blotting. The constant up-regulation of SARM1 in the spinal cord and sciatic nerve (Fig. 1F, 1G) suggested that the SARM1-dependent axonal destruction pathway was relevant in ACR neuropathy.

In order to directly observe the axon damage caused by ACR, we then measured the axon length of N2a cells processed by ACR for different concentrations and different time (Fig. S1C, S1D). The axons of N2a cells were retracted accompanied by swelling, blebbing and fragmentation in ACR-treated groups (Fig. $1 \mathrm{H}$, 1I). The expression of SARM1 was also up-regulated similarly to that in vivo, and it was dose- and time- 
dependent (Fig. 1J). Taken together, both the SARM1-dependent neuropathy and the spatiotemporal pattern of axon degeneration indicated that the SARM1-dependent axon destruction pathway was involved in ACR neuropathy.

\subsection{The up-regulated SARM1 accumulates on mitochondria in ACR neuropathy}

Immunofluorescence was then conducted to determine the intracellular localization of SARM1. As demonstrated in Fig. 2A, SARM1 aggregated into puncta along the nerve fibres, and even translocated in the soma of a motor neurons from rats exposed to ACR for four weeks. Combined with the research on the subcellular localization of SARM1, we speculated that these plaque-like and punctate structures may be linked to the mitochondrial localization of SARM1. Then, we quantified mitochondrial SARM1 by isolating the mitochondrial fraction and conformed that SARM1 was enriched on mitochondria in ACR neuropathy. In the control group, most of the normally expressed SARM1 presented in the mitochondrial fraction. And in the low-dose group, SARM1 in the mitochondria fraction changed significantly, despite that the cytoplasmic level was unchanged (Fig. 2B). Compared with the changes in the cytoplasm, the more obvious alteration was observed in the mitochondria fraction confirming the accumulation of SARM1 on mitochondria (Fig. 2B). The climbed co-localization of SARM1 and PINK1/Parkin, markers of mitophagy, further proved the above conclusion (Fig. 2C, Fig. S2A,). The co-localization of SARM1 and PINK1/Parkin dramatically increased along axons in the low-, medium-, and high-dose group. And evident co-localization in a motor neuron soma was found for the high-dose group. This gathered SARM1 signal, which only presented in axons at lower doses and furtherly arose at the neuro soma at higher doses, was also consistent with the pathological damage of ACR neuropathy that from axon to soma.

Then, the ACR-induced mitochondrial aggregation of SARM1 was verified in vitro. The immunofluorescence of SARM1 presented as dots in ACR treated N2a cells. Co-localization degree of SARM1 with mitochondria-related molecules, e.g. Parkin and DRP1, also markedly increased (Fig. 2D, S2B). Next, we focused on mitochondria to further investigate the biological significance of SARM1 mitochondrial localization in ACR neuropathy.

\subsection{Mitochondrial dynamics are disturbed and mitophagy is activated in ACR neuropathy}

Transmission electron microscopy analysis demonstrated that there were a large number of organelles in the swollen axons from rats exposed to ACR for four weeks, including fragmented mitochondria and some autophagy-related structures (Fig. 3A). The morphological changes of mitochondria in the spinal cord and sciatic nerve were similar. In ACR- treated group, the mitochondria were spherical and elliptical with severely disorganized and swollen cristae, while the mitochondria in the control formed short tubules with a clear sheet-like structure of mitochondrial cristae. Analysis of those images showed that ACR caused an increase in the mitochondrial number, but the length of mitochondria was shortened. And the frequency distribution of mitochondrial length for ACR-treated rats was concentrated in a shorter area (Fig. 3B). Western blotting results were in agreement with the morphological findings, disclosing the 
fragmentation trend of the mitochondrial network. The protein levels of DRP1 and p-DRP (Ser616), which promoted mitochondrial fission, were significantly raised. By contrast, the proteins involved in mitochondrial fusion, e.g. Mfn2 and OPA1, were markedly reduced (Fig. 3C). Furthermore, western blotting also illustrated that PINK1, Parkin, OPTN, NDP52, LC3-区, LC3-区/区, and P62, critical checkpoints for mitophagy, increased dramatically in ACR-intoxicated rats (Fig. 3D-F, Fig. S3A). In addition to these, we also detected lysosomal function-related indicators such as LAMP1 and LAMP2 (Fig. S3B). Fragmented mitochondrial network, increased mitophagy-related proteins and enhanced lysosomal activity preliminarily suggested that the accumulation of mitophagy markers in ACR neuropathy was due to induction of mitophagy rather than blockade of autophagic flux. We further verified this by in vitro experiments.

Firstly, ACR also up-regulated mitophagy-related proteins in N2a cells (Fig. 4A). Secondly, we performed an LC3 turnover experiment to ascertain the alteration of autophagic flux (Fig. 4B). Pre-treatment with

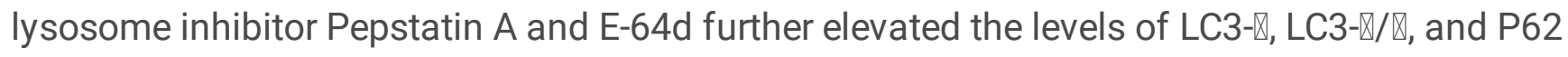
(comparisons labelled by \#) confirming that autophagy was activated in ACR neuropathy with the autophagic flux increased on-rate. Thirdly, the overlap of mitochondrial marker and intracellular acidic organelle marker was consistent with induction of mitophagy through live cell imaging of Mito Tracker Green FM and Lyso Tracker Red DND-99 (Fig. 4C, 4D). The number of lysosomes (red) increased and the mitophagy-lysosome pathway, the colocalization (yellow), was also enhanced in ACR treated N2a cells. Fourthly, the co-localization of mitophagy-related proteins (PINK1 and LC3) and mitochondrial markers (Tom20 and Tim23) increased in ACR-treated cells (Fig. 4E, 4F, Fig. S4). These results fully indicated that ACR activated mitophagy.

\subsection{Rapamycin intervention clears mitochondria accumulated SARM1 and partly alleviates ACR neuropathy}

We have identified mitochondrial accumulation of SARM1 and activation of mitophagy in ACR neuropathy. Considering that SARM1 can bind to and stabilize PINK1 for mitophagy inducing, the mitochondrial localization of SARM1 would likely contribute to its sequestration through increased mitochondrial fission and further clearance through the mitophagy pathway, thereby negatively feedback suppressing axonal degeneration. This hypothesis is in agreement with the results above and the selfrecoverability of ACR poisoning [48].

To test whether this negative feedback scavenging of SARM1 existed or not, we conducted an experiment with RAPA intervention, an autophagy pharmacological activator (Fig. S5A). Rats in the intervention group were addressed in low-dose, pulsing RAPA to intermittently improve basal autophagy and to limit possible adverse effects. Compared with the ACR-intoxication group, abnormal neurobehavioral performances in the RAPA intervention group were delayed, and the severity was lower (Fig. 5A, comparisons labelled by \#). Furthermore, pathological injuries of axons and a motor neurons were improved (Fig. 5B-D), indicating that ACR-induced Wallerian-like degeneration was significantly alleviated following RAPA intervention. Nerve conduction velocity was also obviously ameliorated (Fig. 5E). The 
aggregation of SARM1 on the mitochondria was considerably decreased (Fig. 5F), and mitochondrial dynamics- and mitophagy-related proteins returned to nearly normal levels (Fig. 5F-H Fig. S5B). More importantly, the shape, number, length, and length distribution of mitochondria in the RAPA intervention group recovered with the elimination of mitochondrial SARM1 (Fig. 5I, Fig. S5C). The results suggested that autophagy activator, RAPA, partially rescued the phenotype of ACR neuropathy and also suggested that SARM1 mitochondrial localization and PINK1 mediated mitophagy may be a self-limiting mechanism for axonal degeneration.

\section{Discussion}

Neurotoxicity is the quintessential effect of ACR and Wallerian-like degeneration is typical pathological change of chronic ACR intoxication. In this study, we analyzed ACR-induced programmed axon death in vivo and in vitro, confirming that the SARM1-dependent axonal destruction pathway was associated with peripheral nerve damage in ACR poisoning. Furthermore, we found that the mitochondrial SARM1-PINK1 pathway for mitophagy is one of the molecular mechanisms of autophagy induction by ACR. And mitochondrial localization of SARM1 facilitates its clearance through increased fission and mitophagy, thereby negatively feeding back programmed axonal destruction pathway.

The mitochondrial localization of SARM1 involves in mitochondrial neurites distribution, anoxic degeneration [49] and neuronal survival regulations [50-52]. But some studies prove that deletion of the mitochondrial localization sequence does not alter its ability to promote axon destruction [18]. We consider that these inconsistent results may be ascribed to differences in the type of injury, the dose, and the course of the disease. The axon destruction models of axotomy [17] or chemotherapy-induced peripheral neuropathy, e.g. vincristine, bortezomib and paclitaxel [53,54], induce rapid axonal death with a relatively short latent period, making it difficult to clear rapidly activated SARM1 through the process of mitophagy. Results in this study preliminarily confirmed that the SARM1-dependent axonal destruction pathway is involved in ACR neuropathy. Moreover, the level of NMNAT2 did not decrease (Fig. 1F, G). The relevant multi-omics data also suggest that the NAD + level in the ACR intoxication model is still maintained at a relatively high level (Fold Change of the Control $=0.79, p=0.005$, false discovery rate $(F D R)=1.61 \%)$ [55], instead of depleted by increased SARM1. Although there are axons entered the active degeneration stage and lost, these above indicate that there are axons still in the latent stage of axon destruction in rats subjected to ACR for four weeks. Combined with our results, the mitochondrial localization of SARM1 will help it to be isolated by the mitochondrial network and to be degraded through mitophagy to maintain local axon homeostasis. When the mitochondrial quality control mechanisms are broken down, SARM1 will cause irreversible damage for axon death. Mitochondrial localization of SARM1 contributes to its clearance through the SARM1-PINK1 mitophagy pathway and mitophagy, as the complementary to the coordinated activities of NMNAT2 and SARM1, limits programmed axon death through the clearance of the pro-degenerative factor in ACR peripheral neuropathy.

Mitochondria are dynamic organelles. They are actively recruited to specific cellular locations, fuse and divide continually which serves to intermix the lipids and contents of a population of mitochondria, and 
have dynamic structures under fine quality control conditions [56-59]. At present, increasing evidence support that autophagy is involved in the process of axon degeneration [60-64]. And mitophagy, a specific type of autophagy that selectively degrades defective mitochondria, has received extra attention in energy maintenance for damaged axons. PINK1 is a serine/threonine kinase with an N-terminal mitochondrial targeting sequence and PINK1 dependent pathway is one of the best-studied mitophagy mechanisms [65-68]. Accumulation of PINK1 on the dysfunctional mitochondria can recruit Parkin, OPTN, NDP52 etc., and these binding partners of PINK1, in turn, induce the degradation of the damaged mitochondria through activated mitophagy. Previous studies have found that SARM1 in the mitochondrial outer membrane contributes to the stabilization of PINK1 and mitophagy induction. Therefore, mitochondrial localization of SARM1 will help to sequester SARM1 through the mitochondrial dynamic process, and to the final degradation by mitophagy in ACR peripheral neuropathy. This is consistent with the related results of RAPA intervention.

\section{Conclusion}

Taken together, the study here finds that the up-regulated SARM1 induced by ACR intoxication accumulates on mitochondria with the $\mathrm{N}$-terminal mitochondrial targeting sequence, which stabilizes PINK1 and triggers the mitophagy degradation. Mitophagy clearance of SARM1 is complementary to the coordinated activity of NMNAT2 and SARM1 in ACR neuropathy. Enhancements of autophagic flow with pharmacological methods will promote the clearance of SARM1 and prevent it from breaking down NAD + metabolism, mitochondrial quality control, and other homeostasis mechanisms. Our research preliminarily demonstrated the potential role of mitophagy in ACR-induced toxic peripheral neuropathy. Further elucidating the mechanistic link between mitophagy and SARM1-dependent axonal degeneration will help to develop new strategies for the prevention and treatment of a variety of axon destruction disorders.

\section{Abbreviations}




\begin{tabular}{ll} 
ACR & acrylamide; \\
\hline ARM & armadillo motifs; \\
\hline NAD+ & nicotinamide adenine dinucleotide; \\
\hline NMN & nicotinamide mononucleotide; \\
\hline NMNAT2 & nicotinamide mononucleotide adenyltransferase $2 ;$ \\
\hline PINK1 & PTEN-induced putative kinase 1; \\
\hline RAPA & rapamycin; \\
\hline SAM & sterile a motif; \\
\hline SARM & sterile-a and toll/interleukin 1 receptor motif containing protein 1; \\
\hline TIR & toll/interleukin 1 receptor; \\
\hline TRAF6 & tumor necrosis factor receptor-associated factor 6;
\end{tabular}

\section{Declarations}

\section{Funding}

This work was supported by the National Natural Science Foundation of China (No. 82173552; No. 81673209).

\section{Competing interests}

The authors have no relevant financial or non-financial interests to disclose.

\section{Author contributions}

Shuai Wang, a major contributor in writing the manuscript, performed the examination, analyzed and interpreted the data. Hui Yong, Cuiqin Zhang, Kang Kang, Mingxue Song, Yiyu Yang, Zhengcheng Huang, Shu'e Wang Haotong Ge and Xiulan Zhao contributed to the acquisition of data. Fuyong Song was responsible for conception and design of the work. All authors read and approved the final manuscript.

\section{Data Availability}

The datasets generated during and/or analysed during the current study are available from the corresponding author on reasonable request. 


\section{Ethics approval}

Animals were treated according to the NIH Guide for Care and Use of Laboratory Animals and followed the principles in the "Use of Animals in Toxicology". All protocols were approved by the Institutional Animal Care and Use Committee of Shandong University.

\section{Consent to participate}

Not applicable.

\section{Consent to publish}

Not applicable.

\section{Acknowledgments}

The authors want to thank Translational Medicine Core Facility of Shandong University for consultation and instrument availability that supported this work, Lihua Yu and Wenwen Zheng for their assistance in experiments, Wenhao Yu and Ziqiang Yu for their generous help in data analysis.

\section{References}

1. Waller A (1851) Experiments on the Section of the Glosso-Pharyngeal and Hypoglossal Nerves of the Frog, and Observations of the Alterations Produced Thereby in the Structure of Their Primitive Fibres. Edinb Med Surg J 76(189):369-376

2. Perry VH, Brown MC, Lunn ER, Tree P, Gordon S (1990) Evidence that Very Slow Wallerian Degeneration in C57BL/Ola Mice is an Intrinsic Property of the Peripheral Nerve. Eur J Neurosci 2(9):802-808

3. Perry VH, Brown MC, Lunn ER (1991) Very Slow Retrograde and Wallerian Degeneration in the CNS of C57BL/Ola Mice. Eur J Neurosci 3(1):102-105

4. Lyon MF, Ogunkolade BW, Brown MC, Atherton DJ, Perry VH (1993) A gene affecting Wallerian nerve degeneration maps distally on mouse chromosome 4. Proc Natl Acad Sci USA 90(20):9717-9720

5. Desbois M, Crawley O, Evans PR, Baker ST, Masuho I, Yasuda R et al (2018) PAM forms an atypical SCF ubiquitin ligase complex that ubiquitinates and degrades NMNAT2. J Biol Chem 293(36):13897-13909

6. Babetto E, Beirowski B, Russler EV, Milbrandt J, DiAntonio A (2013) The Phr1 ubiquitin ligase promotes injury-induced axon self-destruction. Cell Rep 3(5):1422-1429 
7. Figley MD, Gu W, Nanson JD, Shi Y, Sasaki Y, Cunnea K et al (2021) SARM1 is a metabolic sensor activated by an increased NMN/NAD + ratio to trigger axon degeneration.Neuron.

8. Essuman K, Summers DW, Sasaki Y, Mao X, DiAntonio A, Milbrandt J (2017) The SARM1 Toll/Interleukin-1 Receptor Domain Possesses Intrinsic NAD(+) Cleavage Activity that Promotes Pathological Axonal Degeneration. Neuron 93(6):1334-43e5

9. Gerdts J, Summers DW, Milbrandt J, DiAntonio A (2016) Axon Self-Destruction: New Links among SARM1, MAPKs, and NAD + Metabolism. Neuron 89(3):449-460

10. Gould SA, Gilley J, Ling K, Jafar-Nejad P, Rigo F, Coleman M (2021) Sarm1 haploinsufficiency or low expression levels after antisense oligonucleotides delay programmed axon degeneration. Cell Rep 37(11):110108

11. Mink M, Fogelgren B, Olszewski K, Maroy P, Csiszar K (2001) A novel human gene (SARM) at chromosome 17q11 encodes a protein with a SAM motif and structural similarity to Armadillo/betacatenin that is conserved in mouse, Drosophila, and Caenorhabditis elegans. Genomics 74(2):234244

12. Meijer AH, Gabby Krens SF, Medina Rodriguez IA, He S, Bitter W, Ewa Snaar-Jagalska B et al (2004) Expression analysis of the Toll-like receptor and TIR domain adaptor families of zebrafish. Mol Immunol 40(11):773-783

13. Couillault C, Pujol N, Reboul J, Sabatier L, Guichou JF, Kohara Y et al (2004) TLR-independent control of innate immunity in Caenorhabditis elegans by the TIR domain adaptor protein TIR-1, an ortholog of human SARM. Nat Immunol 5(5):488-494

14. Belinda LW, Wei WX, Hanh BT, Lei LX, Bow H, Ling DJ (2008) SARM: a novel Toll-like receptor adaptor, is functionally conserved from arthropod to human. Mol Immunol 45(6):1732-1742

15. Panneerselvam P, Singh Laishram P, Ho B, Chen J, Ding Jeak L (2012) Targeting of pro-apoptotic TLR adaptor SARM to mitochondria: definition of the critical region and residues in the signal sequence. Biochem J 442(2):263-271

16. Murata H, Sakaguchi M, Kataoka K, Huh N-H (2013) SARM1 and TRAF6 bind to and stabilize PINK1 on depolarized mitochondria. Mol Biol Cell 24(18):2772-2784

17. Loreto A, Di Stefano M, Gering M, Conforti L (2015) Wallerian Degeneration Is Executed by an NMNSARM1-Dependent Late Ca(2+) Influx but Only Modestly Influenced by Mitochondria. Cell Rep 13(11):2539-2552

18. Gerdts J, Summers DW, Sasaki Y, DiAntonio A, Milbrandt J (2013) Sarm1-mediated axon degeneration requires both SAM and TIR interactions. J Neurosci 33(33):13569-13580

19. Pennisi M, Malaguarnera G, Puglisi V, Vinciguerra L, Vacante M, Malaguarnera M (2013) Neurotoxicity of acrylamide in exposed workers. Int J Environ Res Public Health 10(9):3843-3854

20. Tareke E, Rydberg P, Karlsson P, Eriksson S, Törnqvist M (2002) Analysis of acrylamide, a carcinogen formed in heated foodstuffs. J Agric Food Chem 50(17):4998-5006

21. Schettgen T, Weiss T, Drexler H, Angerer J (2003) A first approach to estimate the internal exposure to acrylamide in smoking and non-smoking adults from Germany. Int J Hyg Environ Health 206(1):9- 
22. Urban M, Kavvadias D, Riedel K, Scherer G, Tricker AR (2006) Urinary mercapturic acids and a hemoglobin adduct for the dosimetry of acrylamide exposure in smokers and nonsmokers. Inhal Toxicol 18(10):831-839

23. Lantz I, Ternité R, Wilkens J, Hoenicke K, Guenther H, van der Stegen GH (2006) Studies on acrylamide levels in roasting, storage and brewing of coffee. Mol Nutr Food Res 50(11):1039-1046

24. He FS, Zhang SL, Wang HL, Li G, Zhang ZM, Li FL et al (1989) Neurological and electroneuromyographic assessment of the adverse effects of acrylamide on occupationally exposed workers. Scand J Work Environ Health 15(2):125-129

25. Deng H, He F, Zhang S, Calleman CJ, Costa LG (1993) Quantitative measurements of vibration threshold in healthy adults and acrylamide workers. Int Arch Occup Environ Health 65(1):53-56

26. Edwards PM, Parker VH (1977) A simple, sensitive, and objective method for early assessment of acrylamide neuropathy in rats. Toxicol Appl Pharmacol 40(3):589-591

27. Kuperman AS (1958) Effects of acrylamide on the central nervous system of the cat. J Pharmacol Exp Ther 123(3):180-192

28. Fullerton PM, Barnes JM (1966) Peripheral neuropathy in rats produced by acrylamide. Br J Ind Med 23(3):210-221

29. Hopkins A (1970) The effect of acrylamide on the peripheral nervous system of the baboon. J Neurol Neurosurg Psychiatry 33(6):805-816

30. Hopkins AP, Gilliatt RW (1971) Motor and sensory nerve conduction velocity in the baboon: normal values and changes during acrylamide neuropathy. J Neurol Neurosurg Psychiatry 34(4):415-426

31. Sumner AJ, Asbury AK (1974) Acrylamide neuropathy: selective vulnerability of sensory fibers. Trans Am Neurol Assoc 99:79-83

32. Sumner AJ, Asbury AK (1975) Physiological studies of the dying-back phenomenon. Muscle stretch afferents in acrylamide neuropathy. Brain 98(1):91-100

33. Erkekoglu P, Baydar T (2014) Acrylamide neurotoxicity. Nutr Neurosci 17(2):49-57

34. LoPachin RM, Barber DS, He D, Das S (2006) Acrylamide inhibits dopamine uptake in rat striatal synaptic vesicles. Toxicol Sci 89(1):224-234

35. Barber DS, Stevens S, LoPachin RM (2007) Proteomic analysis of rat striatal synaptosomes during acrylamide intoxication at a low dose rate. Toxicol Sci 100(1):156-167

36. Ogawa B, Ohishi T, Wang L, Takahashi M, Taniai E, Hayashi H et al (2011) Disruptive neuronal development by acrylamide in the hippocampal dentate hilus after developmental exposure in rats. Arch Toxicol 85(8):987-994

37. Crowe A, Bruelisauer A, Duerr L, Guntz P, Lemaire M (1999) Absorption and Intestinal Metabolism of SDZ-RAD and Rapamycin in Rats. 27:627-6325

38. Rapamycin Confers Neuroprotection Against Aging-Induced Oxidative Stress (2019) Mitochondrial Dysfunction, and Neurodegeneration in Old Rats Through Activation of Autophagy. 22:60-701 
39. Hadamitzky M, Herring A, Kirchhof J, Bendix I, Haight MJ, Keyvani K et al (2018) Repeated Systemic Treatment with Rapamycin Affects Behavior and Amygdala Protein Expression in Rats. Int $\mathrm{J}$ Neuropsychopharmacol 21(6):592-602

40. Clifton GL, Jiang JY, Lyeth BG, Jenkins LW, Hamm RJ, Hayes RL (1991) Marked protection by moderate hypothermia after experimental traumatic brain injury. $\mathrm{J}$ Cereb blood flow metabolism: official J Int Soc Cereb Blood Flow Metabolism 11(1):114-121

41. Monville C, Torres EM, Dunnett SB (2006) Comparison of incremental and accelerating protocols of the rotarod test for the assessment of motor deficits in the 6-OHDA model. J Neurosci Methods 158(2):219-223

42. Youssef AF, Santi BW (1997) Simple Neurobehavioral Functional Observational Battery and Objective Gait Analysis Validation by the Use of Acrylamide and Methanol with a Built-In Recovery Period. Environ Res 73(1):52-62

43. Moser VC, Anthony DC, Sette WF, MacPhail RC (1992) Comparison of subchronic neurotoxicity of 2hydroxyethyl acrylate and acrylamide in rats. Fundam Appl Toxicol 18(3):343-352

44. LoPachin RM, Ross JF, Reid ML, Das S, Mansukhani S, Lehning EJ (2002) Neurological Evaluation of Toxic Axonopathies in Rats: Acrylamide and 2,5-Hexanedione. Neurotoxicology 23(1):95-110

45. Takeuchi Y, Ono Y, Hisanaga N, Kitoh J, Sugiura Y (1980) A comparative study on the neurotoxicity of $\mathrm{n}$-pentane, $\mathrm{n}$-hexane, and $\mathrm{n}$-heptane in the rat. $\mathrm{Br} \mathrm{J}$ Ind Med 37(3):241-247

46. Lima RT, Sousa D, Paiva AM, Palmeira A, Barbosa J, Pedro M et al (2016) Modulation of Autophagy by a Thioxanthone Decreases the Viability of Melanoma Cells.Molecules (Basel, Switzerland). ; 21(10)

47. Kong FJ, Wu JH, Sun SY, Zhou JQ (2017) The endoplasmic reticulum stress/autophagy pathway is involved in cholesterol-induced pancreatic $\beta$-cell injury. Sci Rep 7:44746

48. Burek JD, Albee RR, Beyer JE, Bell TJ, Carreon RM, Morden DC et al (1980) Subchronic toxicity of acrylamide administered to rats in the drinking water followed by up to 144 days of recovery. $J$ Environ Pathol Toxicol 4(5-6):157-182

49. Hayakawa T, Kato K, Hayakawa R, Hisamoto N, Matsumoto K, Takeda K et al (2011) Regulation of anoxic death in Caenorhabditis elegans by mammalian apoptosis signal-regulating kinase (ASK) family proteins. Genetics 187(3):785-792

50. Kim Y, Zhou P, Qian L, Chuang J-Z, Lee J, Li C et al (2007) MyD88-5 links mitochondria, microtubules, and JNK3 in neurons and regulates neuronal survival. J Exp Med 204(9):2063-2074

51. Mukherjee P, Winkler CW, Taylor KG, Woods TA, Nair V, Khan BA et al (1950) SARM1, Not MyD88, Mediates TLR7/TLR9-Induced Apoptosis in Neurons. Journal of immunology (Baltimore, Md: 2015;195(10):4913-21

52. Killackey SA, Rahman MA, Soares F, Zhang AB, Abdel-Nour M, Philpott DJ et al (2019) The mitochondrial Nod-like receptor NLRX1 modifies apoptosis through SARM1. Mol Cell Biochem 453(1):187-196 
53. Geisler S, Doan RA, Cheng GC, Cetinkaya-Fisgin A, Huang SX, Höke A et al (2019) Vincristine and bortezomib use distinct upstream mechanisms to activate a common SARM1-dependent axon degeneration program. JCI Insight 4(17):e129920

54. Bosanac T, Hughes RO, Engber T, Devraj R, Brearley A, Danker K et al (2021) Pharmacological SARM1 inhibition protects axon structure and function in paclitaxel-induced peripheral neuropathy.Brain.

55. Prats E, Gómez-Canela C, Ben-Lulu S, Ziv T, Padrós F, Tornero D et al (2017) Modelling acrylamide acute neurotoxicity in zebrafish larvae. Sci Rep 7(1):13952

56. MacAskill AF, Kittler JT (2010) Control of mitochondrial transport and localization in neurons. Trends Cell Biol 20(2):102-112

57. Chen H, Chan DC (2009) Mitochondrial dynamics-fusion, fission, movement, and mitophagy-in neurodegenerative diseases. Hum Mol Genet 18(R2):R169-R176

58. Schon EA, Przedborski S (2011) Mitochondria: the next (neurode)generation. Neuron 70(6):10331053

59. Chan DC (2006) Mitochondria: dynamic organelles in disease, aging, and development. Cell 125(7):1241-1252

60. Ravikumar B, Vacher C, Berger Z, Davies JE, Luo S, Oroz LG et al (2004) Inhibition of mTOR induces autophagy and reduces toxicity of polyglutamine expansions in fly and mouse models of Huntington disease. Nat Genet 36(6):585-595

61. Pickford F, Masliah E, Britschgi M, Lucin K, Narasimhan R, Jaeger PA et al (2008) The autophagyrelated protein beclin 1 shows reduced expression in early Alzheimer disease and regulates amyloid beta accumulation in mice. J Clin Invest 118(6):2190-2199

62. Yang Y, Chen S, Zhang J, Li C, Sun Y, Zhang L et al (2014) Stimulation of autophagy prevents amyloid- $\beta$ peptide-induced neuritic degeneration in PC12 cells. J Alzheimer's disease: JAD 40(4):929-939

63. Clarke JP, Mearow K (2016) Autophagy inhibition in endogenous and nutrient-deprived conditions reduces dorsal root ganglia neuron survival and neurite growth in vitro. J Neurosci Res 94(7):653670

64. Wang Y, Song M, Song F (2018) Neuronal autophagy and axon degeneration. Cell Mol Life Sci 75(13):2389-2406

65. Kim I, Rodriguez-Enriquez S, Lemasters JJ (2007) Selective degradation of mitochondria by mitophagy. Arch Biochem Biophys 462(2):245-253

66. Han S, Jeong YY, Sheshadri P, Su X, Cai Q (2020) Mitophagy regulates integrity of mitochondria at synapses and is critical for synaptic maintenance. EMBO Rep 21(9):e49801

67. Kim I, Lemasters JJ (2011) Mitophagy selectively degrades individual damaged mitochondria after photoirradiation. Antioxid Redox Signal 14(10):1919-1928

68. Pollock L, Jardine J, Urbé S, Clague MJ (2021) The PINK1 repertoire: Not just a one trick pony. BioEssays 43(11):e2100168 
Figures
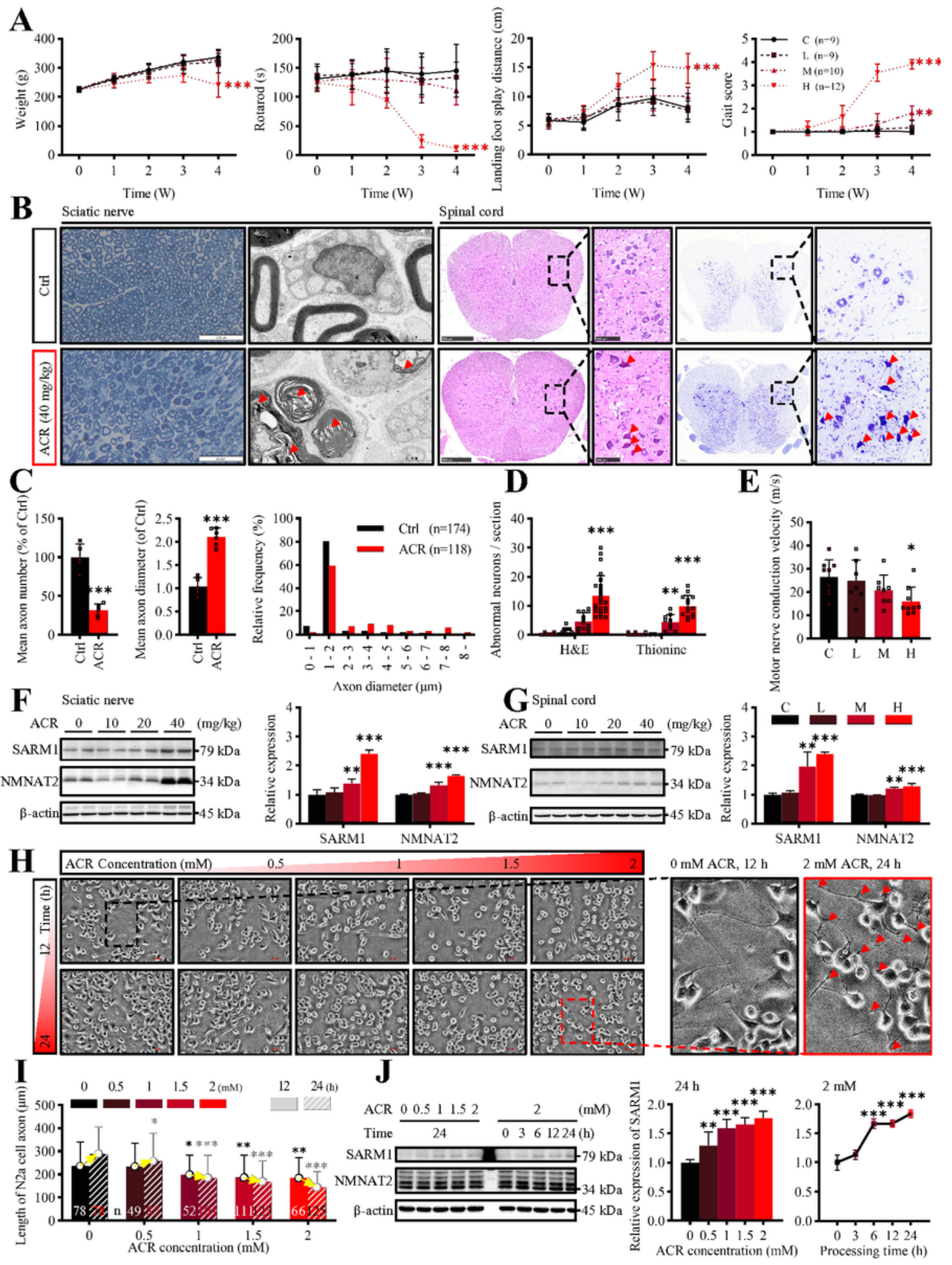

Figure 1

SARM1-dependent axonal destruction pathway is involved in ACR neuropathy. (A) Body weight and neurobehavioral performances of rats subjected to ACR for four weeks. Panels from left to right are weight and neurobehavioral indicators, that are, rotarod, landing foot splay distance, and gait score. (B) 
Axon damage and neuronal morphology changes in rats subjected to ACR for four weeks. Panels from left to right are representative images for Toluidine blue staining, transmission electron microscopy of sciatic nerve cross-sections, H\&E and Thionine staining of spinal cord cross-sections. Red arrowheads in the transmission electron microscope images indicate demyelinated axons. Red arrowheads in the spinal cord images indicate cytoplasmic hyperchromatic a motor neurons. (C) Quantification of axon number, axon diameter, and distribution of axon diameter from transmission electron microscopy images of sciatic nerve cross-sections. (D) Quantification of a motor neurons with hyperchromatic cytoplasm. (E) Motor nerve conduction velocity of rats subjected to ACR for four weeks. (F) Western blotting analysis of Wallerian degeneration-related proteins in the sciatic nerve and $(G)$ in the spinal cord from rats subjected to ACR for four weeks. $n=6$. $(H)$ Representative images and axon length measurement results $(I)$ of N2a cells processed by ACR for different concentrations and different time. The images shown here are faded and contrast strengthened to increase the accuracy of the measurement. Adjustments are applied to the entire image. Red arrowheads indicate the damaged axons that are accompanied by swelling, blebbing, and fragmentation. $(\mathrm{J})$ Western blotting analysis of Wallerian degeneration-related proteins in N2a cells with ACR treated for different concentrations and different time. $n=6 .{ }^{*}, p<0.05 ; * \star, p<0.01 ; * \star \star, p<0.001$ compared to corresponding controls. 

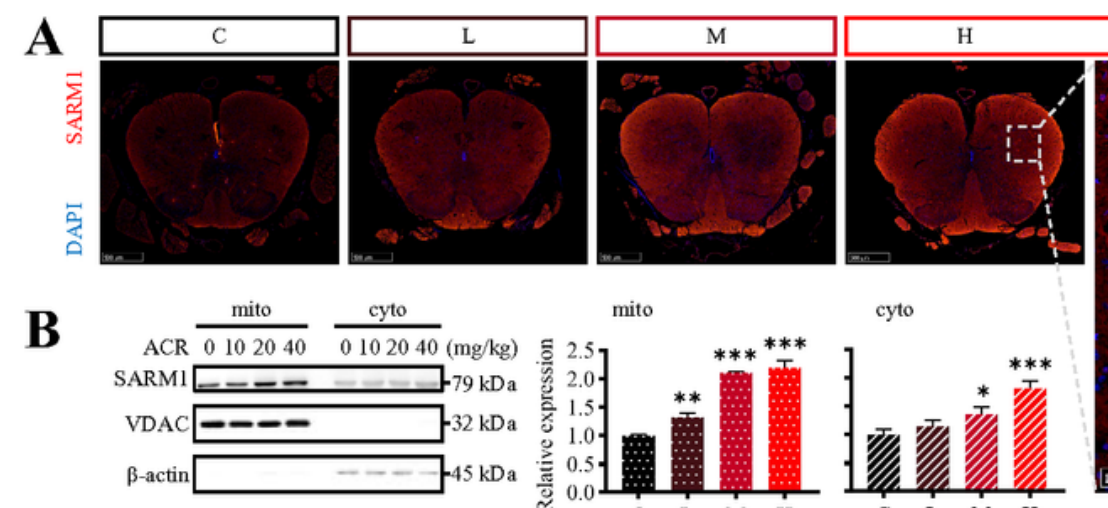

mito
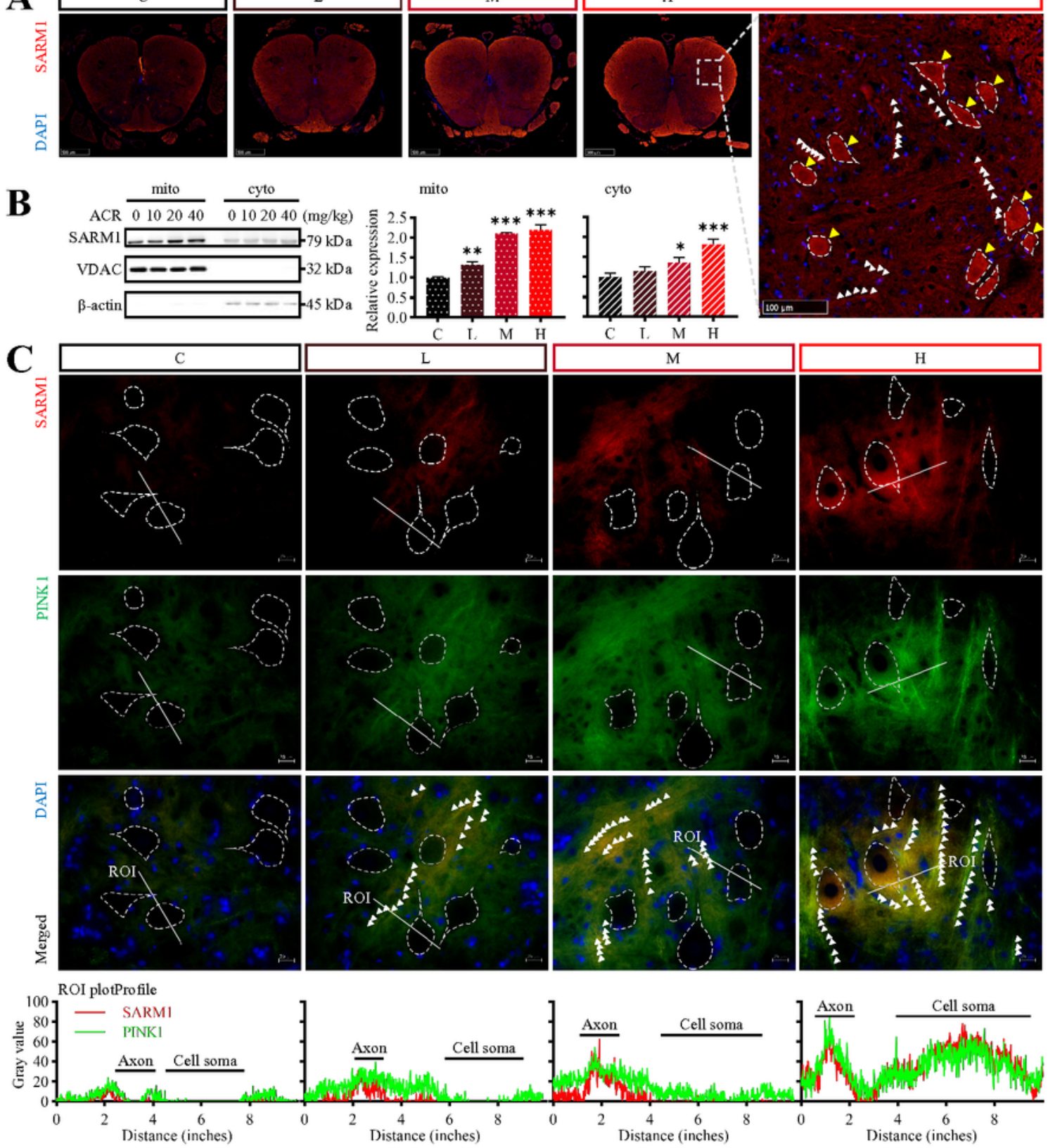

D

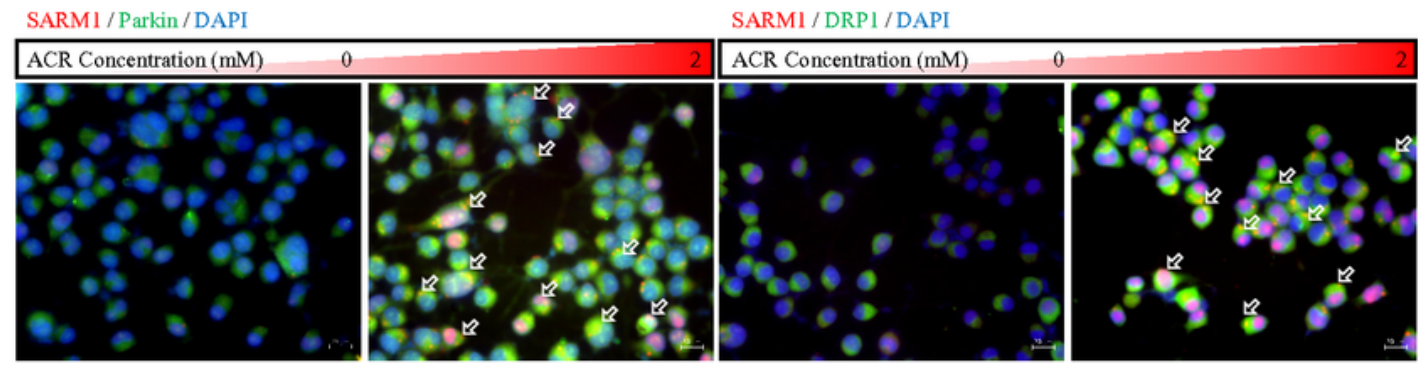

Figure 2

Up-regulated SARM1 accumulates on mitochondria in ACR neuropathy. (A) Representative immunofluorescence images of SARM1 in the spinal cord cross-sections from rats subjected to ACR for four weeks. In the detail display, yellow and white arrowheads refer to the gathered SARM1 in the cell soma and axon, respectively. White dotted lines outline a motor neurons. (B) Western blotting analysis of SARM1. The mitochondrial fraction and the cytoplasmic fraction of the same sample are loaded (40 $\mu \mathrm{g}$ 
per channel) in the same gel for immunoblotting. And sequentially incubate with primary anti-SARM1, anti-VDAC, anti- $\beta$-actin antibodies. VDAC and $\beta$-actin are used for internal control and homogenization of mitochondrial fractions and cytoplasmic fractions, respectively. $n=8$. (C) Immunofluorescence of SARM1 (red) and PINK1 (green) in spinal cord cross-sections from rats subjected to ACR for four weeks. White dotted lines outline a motor neurons and white solid lines are the region of interest (ROI) for gray intensity analysis of red and green channels. White arrowheads point out points where red and green channels colocalize along axons. Scale bar, $20 \mu \mathrm{m}$. (D) Immunofluorescence of SARM1 (red) and Parkin (green), SARM1 (red) and DRP1 (green) in N2a cells with ACR treated for $24 \mathrm{~h}$. The hollow white arrows indicate the punctate-accumulated SARM1. Scale bar, $20 \mu \mathrm{m} .{ }^{*}, p<0.05 ;{ }^{* *}, p<0.01 ; * \star *, p<0.001$ compared to corresponding controls. 
A
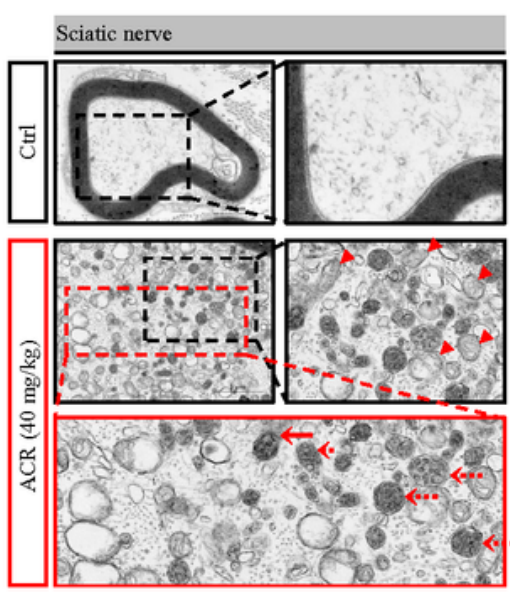
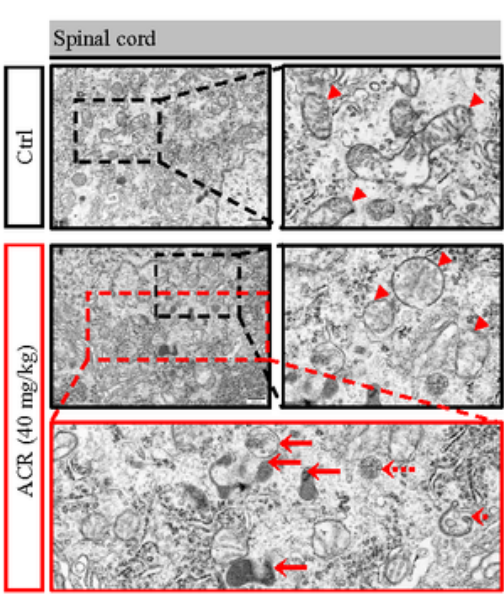

B

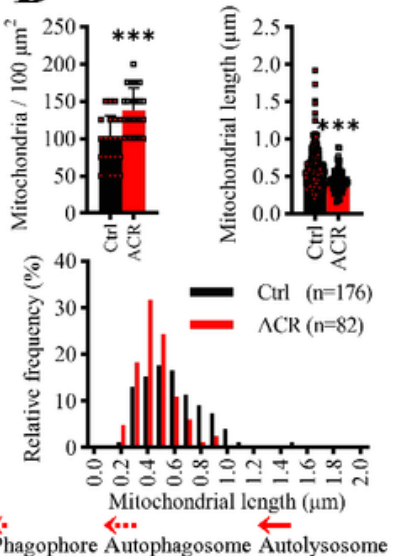

C ACR $\stackrel{0}{\underline{10}} \underline{20} \underline{\underline{40}}(\mathrm{mg} / \mathrm{kg})$

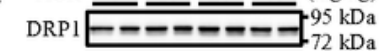
p-DRP ${ }^{s e 616}$ $\mathrm{Mfn} 2-------76 \mathrm{kDa}$ OPAI $E======-72 \mathrm{kDa}$

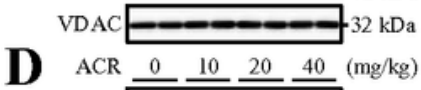
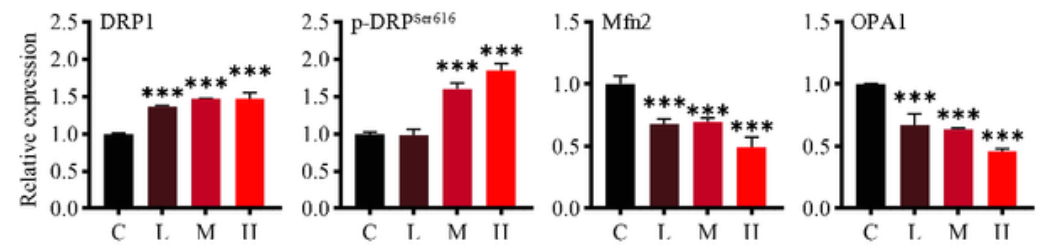

PINK I

Parkin - - $50 \mathrm{kDa}$ OPTN E- $66 \mathrm{kDa}$ NDP52 ------ $52 \mathrm{kDa}$ VDACE- 32 kDa
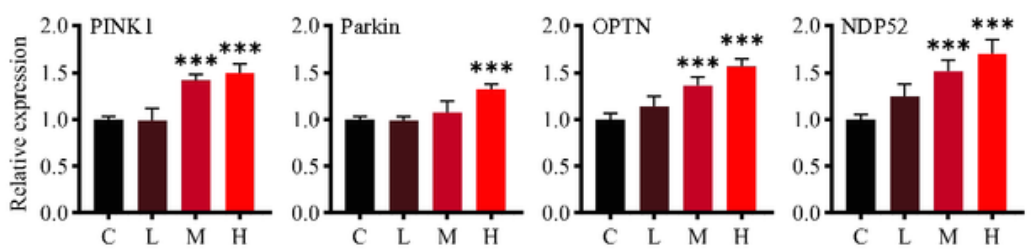

E ACR $0 \underline{10} \underline{\underline{20}} \underline{\underline{40}}(\mathrm{mg} / \mathrm{kg})$ LC3-II $=-1=-18 \mathrm{kDa}$

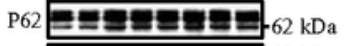
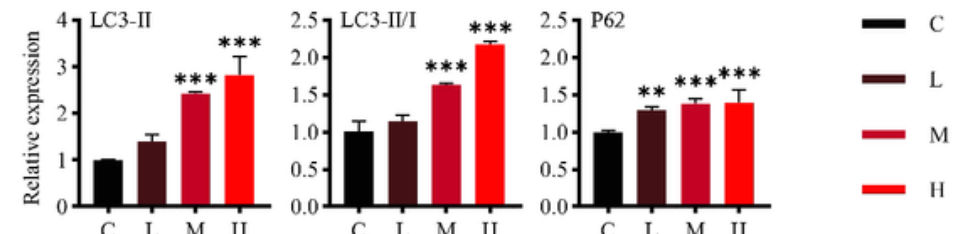

$\mathbf{F}$
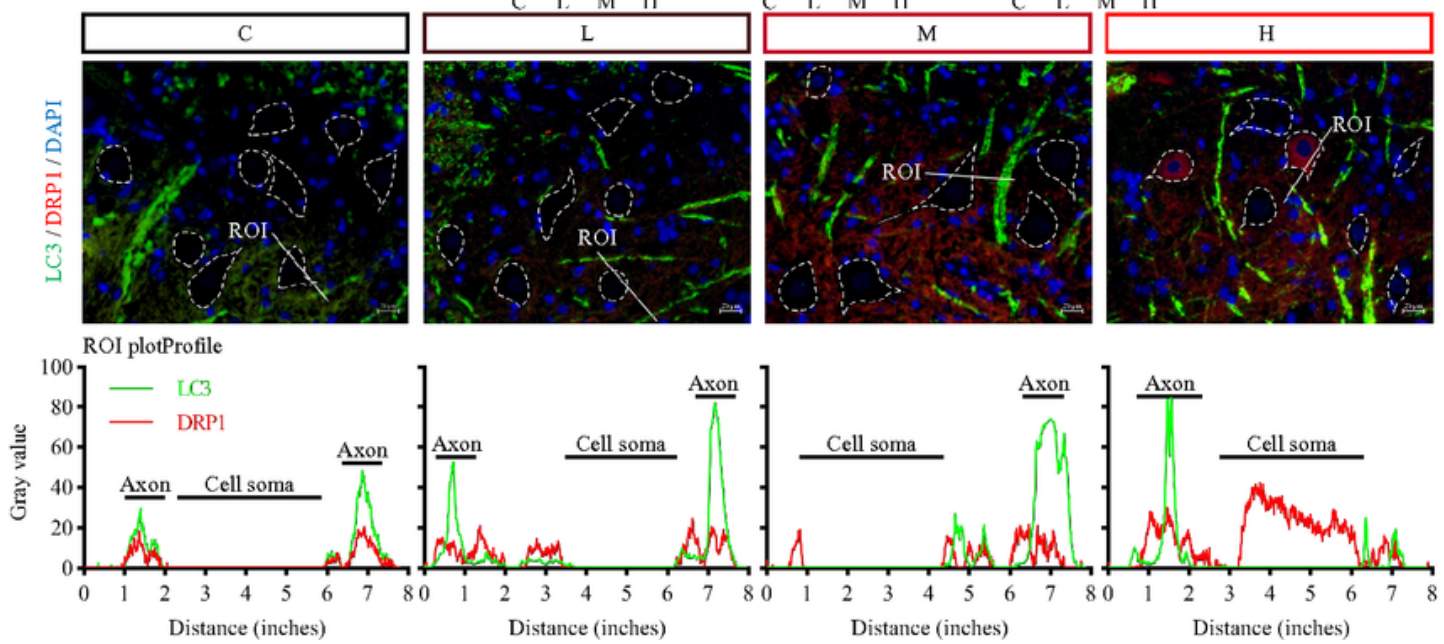

Figure 3

Mitochondrial dynamics are disturbed and mitophagy-related proteins are upregulated in ACR

neuropathy. (A) Representative transmission electron microscopy images of the sciatic nerve and the spinal cord from rats subjected to ACR for four weeks. The black and red dotted boxes show the ultrastructure of mitochondria and autophagy-related vesicles, respectively. Red arrowheads in the black boxes point to mitochondria. The lines of the red arrows in the red box that change from short to long and 
from virtual to solid, indicate the different stages of nucleation and elongation (phagophore), docking and fusion (autophagosome), and degradation (autolysosome) of autophagy-related vesicles. (B) Quantification of mitochondrial number, mitochondrial length, and distribution of mitochondrial length in the spinal cord of rats subjected to ACR for four weeks. (C) Western blotting analysis of mitochondrial dynamic- (D) mitophagy- and (E) autophagy-related proteins in the spinal cord from rats subjected to ACR for four weeks. $n=6$. (F) Immunofluorescence of LC3 (green) and DRP1 (red) in the spinal cord crosssections from rats subjected to ACR for four weeks. White dotted lines outline a motor neurons and white solid lines are the region of interest (ROI) for gray intensity analysis of red and green channels. Scale bar, $20 \mu \mathrm{m} . *, p<0.05 ; * \star, p<0.01 ; * \star \star, p<0.001$ compared to corresponding controls. 
Time 24 (h)

PINK $1 \square 63 \mathrm{kDa}$

Parkin $=-250 \mathrm{kDa}$

VDAC $---132 \mathrm{kDa}$
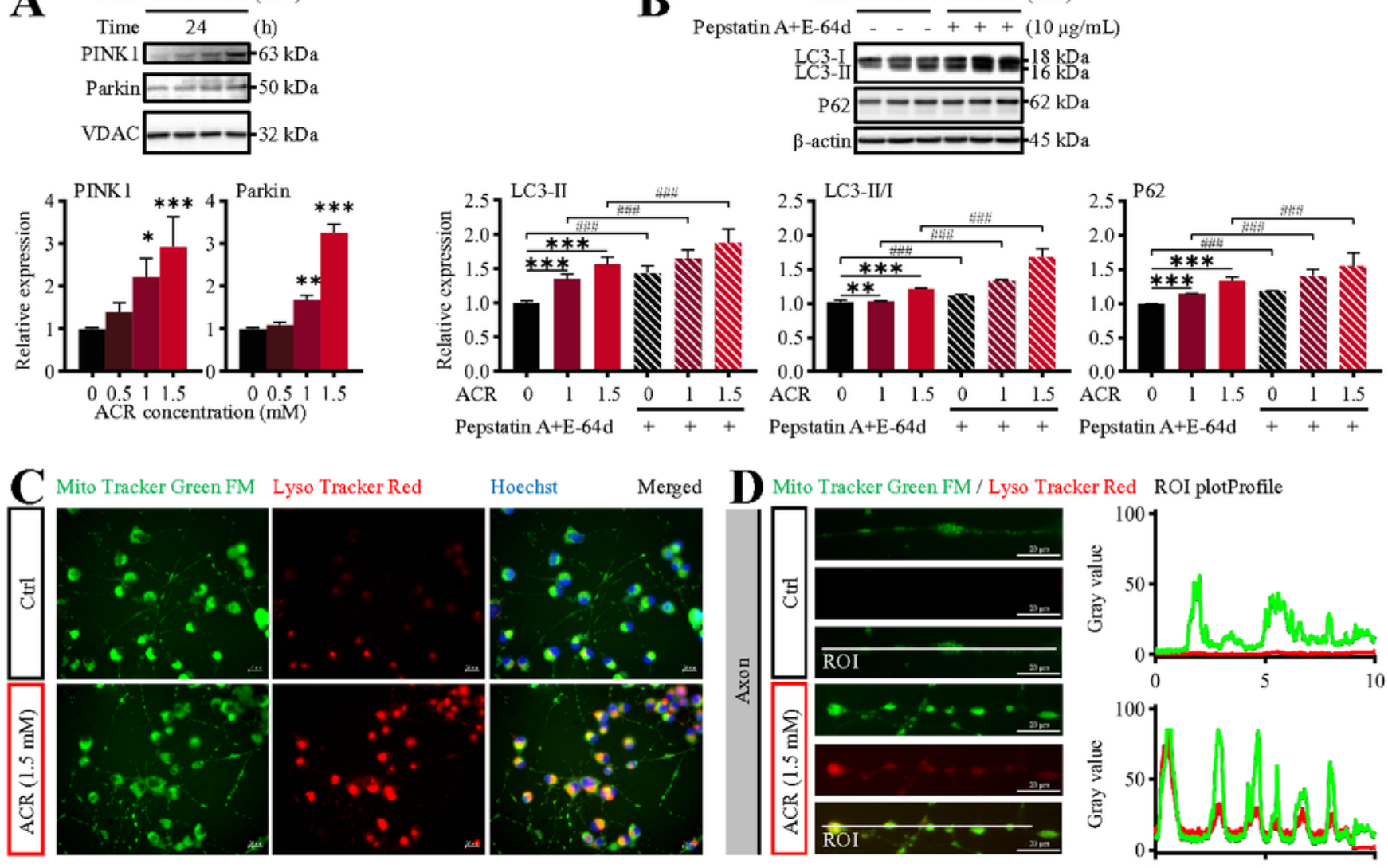

D

Mito Tracker Green FM / Lyso Tracker Red ROI plotProfile
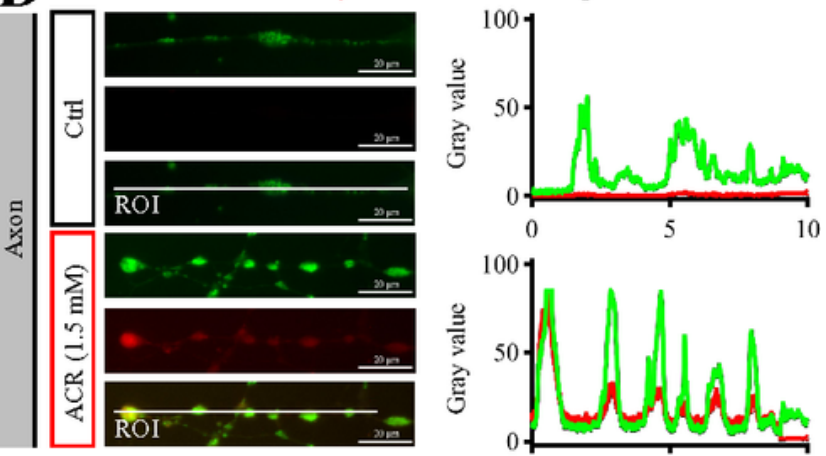

$\mathbf{E}$
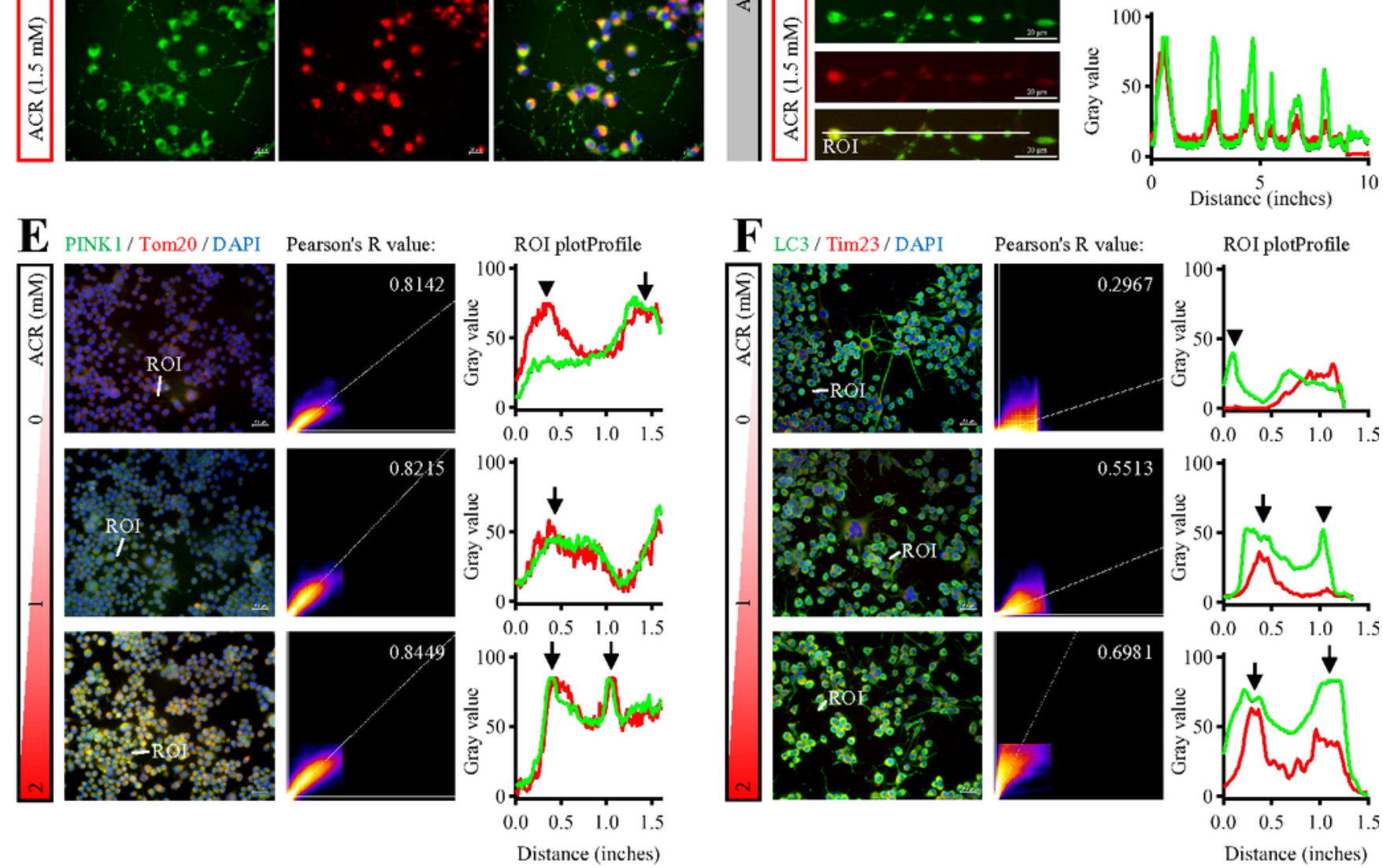

Distance (inches)

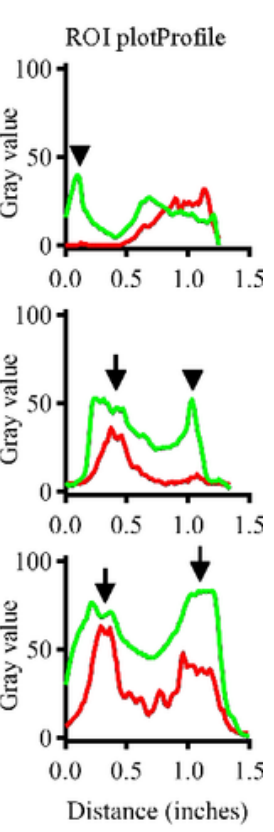

Figure 4

Mitophagy is activated in ACR neuropathy. (A) Western blotting analysis of mitophagy-related proteins in ACR processed N2a cells. $n=6$. (B) Western blotting analysis of LC3 turnover experiment. $n=8$. (C) Live cell imaging of N2a cells with $1.5 \mathrm{mM}$ ACR treated for $24 \mathrm{~h}$ or not. Mito Tracker Green FM and Lyso Tracker Red DND-99 are applied to visualize mitochondria and lysosomes, respectively. Hoechst 33342 was used to stain nuclei. Scale bar, $20 \mu \mathrm{m}$. (D) Representative images of axonal details in C. White solid lines are 
the region of interest (ROI) for gray intensity analysis of red and green channels. Scale bar, $20 \mu \mathrm{m}$. (E) Immunofluorescence of PINK1 (green) and Tom20 (red), (F) LC3 (green) and Tim23 (red) in N2a cells with ACR treated for $24 \mathrm{~h}$. White solid lines are the region of interest (ROI) for gray intensity analysis of red and green channels. Black arrows in ROI plotProfiles indicate overlapped points, and black arrowheads indicate non-co-located points. The calculation of the Pearson's $\mathrm{R}$ value is based on the entire image, and the scatterplot is placed to the right of the corresponding image. Scale bar, $50 \mu \mathrm{m} .{ }^{*}, \mathrm{p}<0.05 ;{ }^{*}, \mathrm{p}<0.01$; $\star \star \star, ~ p<0.001$ compared to corresponding controls. \#, $p<0.05$; \#\#, $p<0.01 ; \# \# \#, p<0.001$, comparison between groups with Pepstatin A and E-64d intervention or not. 

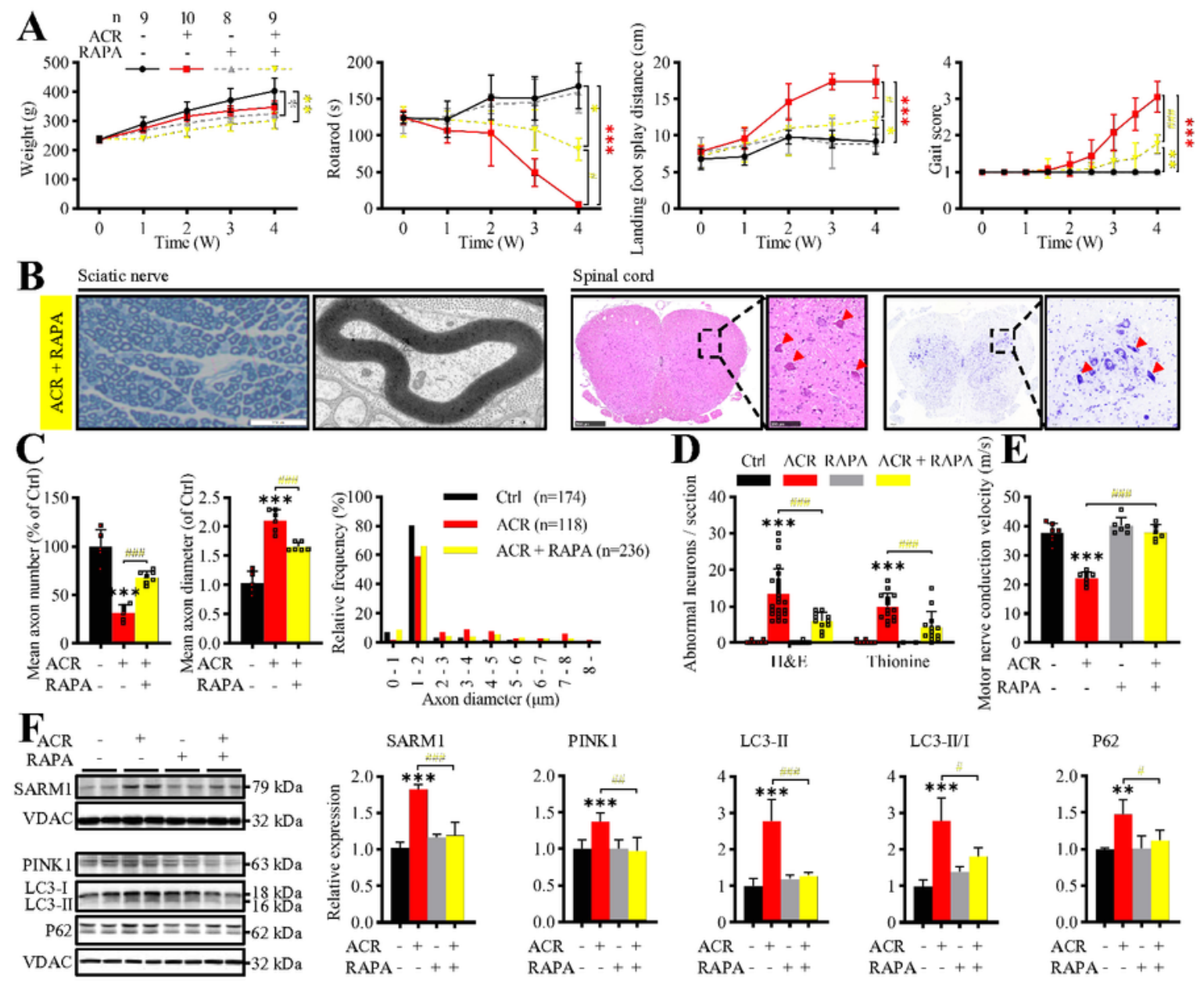

PINK I
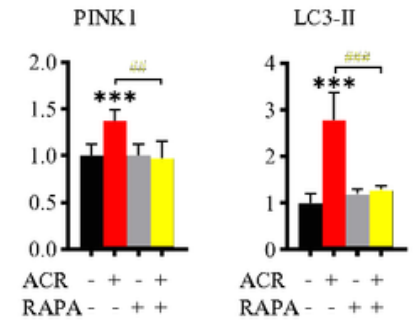

LC3-II/I

P62

G

HSARMI
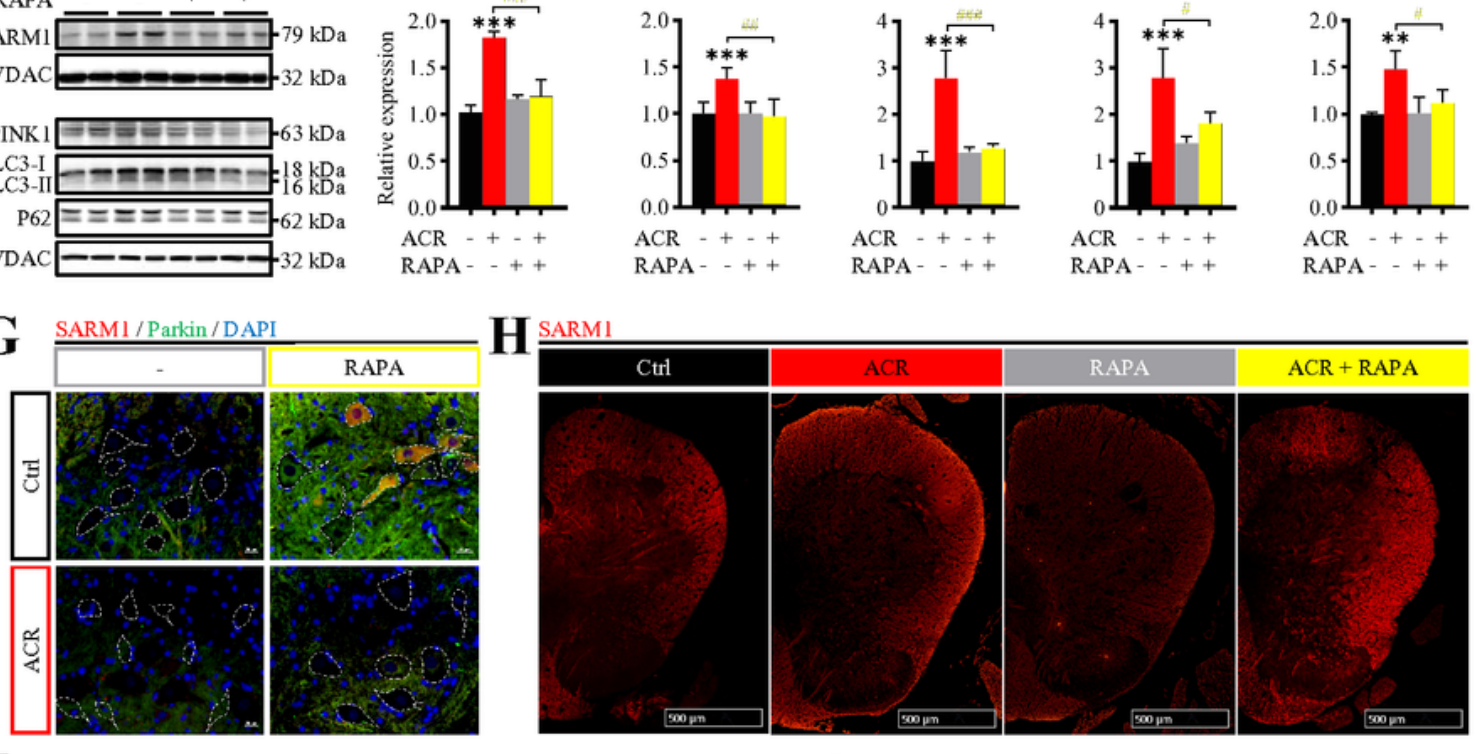

$\mathrm{ACR}+\mathrm{RAPA}$
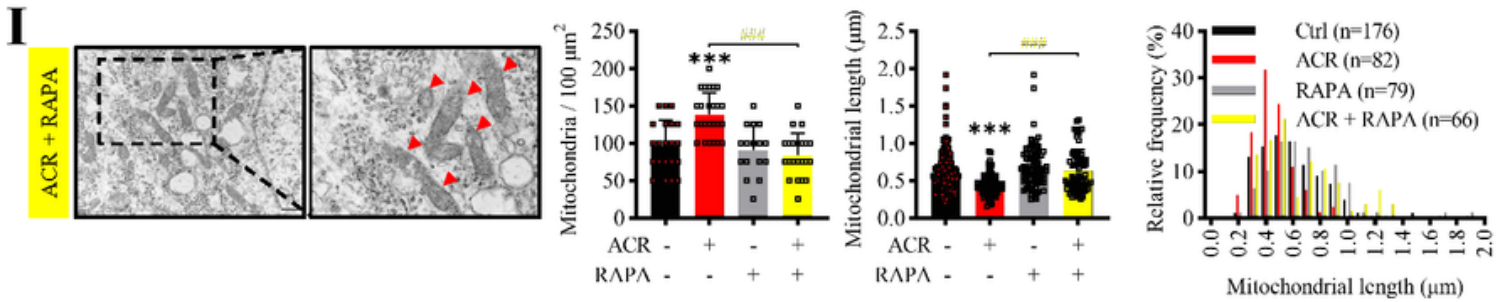

Figure 5

Rapamycin limits the mitochondrial accumulation of SARM1 and rescues the phenotype of ACR poisoning. (A) Body weight and neurobehavioral performances of rats. Panels from left to right are weight and neurobehavioral indicators, that are, rotarod, landing foot splay distance, and gait score. (B) Axon damage and neuronal morphology changes in rats with RAPA intervened for four weeks. Corresponds to Fig. 1B. (C) Quantification of axon number, axon diameter, and distribution of axon 
diameter in the spinal cord of rats with RAPA intervened for four weeks. Corresponds to Fig. 1C. (D) Quantification of a motor neurons with abnormal morphological changes. Corresponds to Fig. 1D. (E) Motor nerve conduction velocity of rats with RAPA intervened or not. (F) Western blotting analysis of SARM1 and mitophagy-related proteins in the spinal cord mitochondrial fractions. $n=6$. $(G)$ Immunofluorescence of SARM1 (red) and Parkin (green) and (H) representative immunofluorescence images of SARM1 in the spinal cord cross-sections from rats with RAPA intervened for four weeks. Corresponds to Fig. S2A and Fig. 2A, respectively. (I) Representative transmission electron microscopy images of the spinal cord from rats with RAPA intervened or not. The black dotted box shows the mitochondria ultrastructure. Histograms on the right are quantification of mitochondrial number, mitochondrial length, and distribution of mitochondrial length in the spinal cord of rats with RAPA intervened. Corresponds to Fig. 3A, 3B. *, $p<0.05 ; * \star, p<0.01 ; * \star \star, p<0.001$ compared to corresponding controls. \#, $\mathrm{p}<0.05 ; \# \#, \mathrm{p}<0.01 ; \# \# \#, \mathrm{p}<0.001$, comparison between groups with RAPA intervention or not.

\section{Supplementary Files}

This is a list of supplementary files associated with this preprint. Click to download.

- Supplementaryfile1.Reagent.xlsx

- Supplementaryfile2.Supplementaryfigures.docx

- GraphicalAbstract.pdf 